\title{
TIME-DELAY OPERATORS IN SEMICLASSICAL LIMIT. II. SHORT-RANGE POTENTIALS
}

\author{
XUE-PING WANG
}

\begin{abstract}
This work is a continuation of [27]. We prove that quantum timedelay operator localized in a nontrapping energy interval is in fact an $h$-pseudodifferential operator with the $h$-principal symbol given by the classical timedelay function composed with the incoming wave operator in classical mechanics. The classical limit of time-delay operator is also given.
\end{abstract}

\section{INTRODUCTION}

Let $H_{0}^{h}=-h^{2} \Delta, H^{h}=H_{0}^{h}+V(x)$, where $\left.\left.h \in\right] 0, h_{0}\right]$ is a small parameter and $V$ is a real, smooth function on $\mathbf{R}^{n}$ satisfying, for some $\varepsilon_{0}>0$,

$$
\left|\partial_{x}^{\alpha} V(x)\right| \leq c_{\alpha}\langle x\rangle^{-1-|\alpha|-\varepsilon_{0}}, \quad x \in \mathbf{R}^{n}, \alpha \in \mathbf{N}^{n} .
$$

Here $\langle x\rangle=\left(1+|x|^{2}\right)^{1 / 2}$. Put $U_{0}(t, h)=\exp \left(i h^{-1} t H_{0}^{h}\right)$ and $U(t, h)=$ $\exp \left(-i h^{-1} t H^{h}\right)$. Define the wave operators $W_{ \pm}(h)$ by

$$
W_{ \pm}(h)=\underset{t \rightarrow \pm \infty}{s-\lim _{i}} U(t, h)^{*} U_{0}(t, h), \quad \text { in } L^{2}\left(\mathbf{R}^{n}\right) .
$$

Let $S(h)=W_{+}(h)^{*} W_{-}(h)$ be the scattering operator. Then it is known (cf. $[1,2,13,23-24]$, and references cited therein) that the time-delay operator for the scattering process $\left(H_{0}^{h}, H^{h}\right)$, defined by taking the large space limit of the difference between sojourn times of the free and interacting particles, exists and is given by:

$$
H_{0}^{h} T(h)=\frac{1}{2} S(h)^{*}[S(h), A(h)], \quad A(h)=\frac{h}{2}\left(x \cdot D_{x}+D_{x} \cdot x\right) .
$$

Equation (1.2) makes sense on the set $D=\left\{f \in L^{2}\left(R^{n}\right) ; \hat{f} \in C_{0}\left(R^{n} \backslash 0\right)\right\}$. In classical scattering theory, there is a time-delay function defined via sojourn times of classical particles (see [11]). Consider the Hamilton system:

$$
\begin{cases}\dot{q}(t)=2 p(t), & q(0)=x, \\ \dot{p}(t)=-\nabla V(q(t)), & p(0)=\xi .\end{cases}
$$

Received by the editors September 27, 1988 and, in revised form, December 15, 1988.

1980 Mathematics Subject Classification (1985 Revision). Primary 35P25, 35J10; Secondary $35 \mathrm{~S} 05,81 \mathrm{C} 05$. 
If $(q(t ; x, \xi), p(t ; x, \xi))$ is a scattering trajectory, i.e., $\lim |q(t ; x, \xi)|=+\infty$, then the classical time-delay function $\tau$ can be defined at $(x, \xi)$ and is equal to:

$$
\tau(x, \xi)=\frac{1}{2 E}\left\{\lim _{t \rightarrow-\infty}(q(t)-2 t p(t)) \cdot p(t)-\lim _{t \rightarrow+\infty}(q(t)-2 t p(t)) \cdot p(t)\right\}
$$

$E=|\xi|^{2}+V(x)$. In this paper, we use the following nontrapping assumption to assure $\tau$ to be defined. Let $J \subset] 0,+\infty[$ be an open interval. We shall call $J$ an interval of nontrapping energy, if for every subinterval $I \subset \subset J$, and for every $R>0$, there exists $t_{0}>0$ such that

$$
|q(t ; x, \xi)|>R, \quad \text { for }|t| \geq t_{0}
$$

and for $(x, \xi) \in p^{-1}(I)$ with $|x|<R$. Here $p(x, \xi)=|\xi|^{2}+V(x)$. From our earlier works, it is clear that the nontrapping condition is important in studying semiclassical propagation properties of scattering states for $H^{h}$. See $[25,26]$.

Before stating the main results of this work, let us introduce some notations. For $\varepsilon \in] 0,2[, d>0, R>0$, we define:

$$
\Omega_{ \pm}(\varepsilon, d, R)=\left\{(x, \xi) \in R^{2 n} ; \pm \widehat{x} \cdot \widehat{\xi}>-1+\varepsilon,|\xi|^{2}>d,|x|>R\right\} .
$$

Here $\hat{x}=x /|x|$ and $\widehat{\xi}=\xi /|\xi|$. Put $\Omega(\varepsilon, d, R)=\Omega_{ \pm}(\varepsilon, d, R) \cap \Omega_{-}(\varepsilon, d, R)$. For $m \in R$, introduce the class of symbols on $R^{2 n}$ :

$$
S^{m}=\left\{a \in C^{\infty}\left(\mathbf{R}^{2 n}\right) ;\left|\partial_{x}^{\alpha} \partial_{\xi}^{\beta} a(x, \xi)\right| \leq C_{\alpha \beta}\langle x\rangle^{m-|\alpha|}\right\} .
$$

Put $S_{ \pm}^{m}(\varepsilon, d, R)=\left\{a \in S^{m} ; \operatorname{supp} a \subset \Omega_{ \pm}(\varepsilon, d, R)\right\}$ and $S_{ \pm}^{m}=\bigcup S_{ \pm}^{m}(\varepsilon, d, R)$, where the union is taken over $\varepsilon \in] 0,2\left[, d>0, R>0\right.$. If $a(h) \in C^{\infty}\left(R^{2 n}\right)$ has an asymptotic expansion of the form:

$$
a(h) \sim \sum_{j=0}^{\infty} h^{N+j} a_{j}, \quad a_{j} \in S^{m-j},
$$

we shall just write: $a(h) \in S^{m, N}$. Similarly, we can define the elements for $S_{ \pm}^{m, N}$. For a symbol $a(h)$, we define an $h$-pseudodifferential operator $(h-\psi \mathrm{DO})$ by

$$
\begin{aligned}
& a(x, h D ; h) u(x)=(2 \pi h)^{-n} \iint e^{i(x-y) \cdot \xi / h} a(x, \xi ; h) u(y) d y d \xi, \\
& u \in \mathscr{S}\left(R^{n}\right) .
\end{aligned}
$$

In the following, we shall not distinguish an $h-\psi \mathrm{DO}$ from an $h$-admissible operator in the sense of Helffer and Robert [4], which is, by definition, an $h$ $\psi \mathrm{DO}$ modulo an error of order $O\left(h^{\infty}\right)$ in $\mathscr{L}\left(L^{2}\right)$. The main results of this paper are the following. 
Theorem 1. Assume that $V$ satisfies (1.1) and $J$ is an interval of nontrapping energy. Let $a, b \in C_{0}^{\infty}\left(\mathbf{R}_{x}^{n}\right)$ or $a, b \in S_{+}^{0} \cap S_{-}^{0}$. In the former case, we shall write: $a(x, \xi)=a(x), \forall \xi \in R^{n}$, etc. Let $f \in C_{0}^{\infty}(J)$. Then $a(x, h D) f\left(H_{0}^{h}\right) T(h) b(x, h D)$ is an $h-\psi D O$ of order zero. More precisely, there exists $t(h) \in S^{0,0}, t(h) \sim \sum_{j=0}^{\infty} h^{j} t_{j}$, with

$$
\left\|a(x, h D) f\left(H_{0}^{h}\right) T(h) b(x, h D)-t(x, h D ; h)\right\|_{\mathscr{L}\left(L^{2}\right)}=O\left(h^{\infty}\right), \quad h \rightarrow 0_{+} .
$$

Moreover, $t_{0}(x, \xi)=a(x, \xi) b(x, \xi) f\left(|\xi|^{2}\right) \tau \circ \Omega_{-}^{\mathrm{cl}}(x, \xi)$. Here $\tau$ is the classical time-delay function and $\Omega_{-}^{\mathrm{cl}}$ is the incoming wave operator in classical mechanics defined by

$$
\Omega_{-}^{\mathrm{cl}}(x, \xi)=\lim _{t \rightarrow+\infty} \phi^{-t} \circ \phi_{0}^{t}(x, \xi), \quad \xi \neq 0 .
$$

Here $\phi^{t}=(q(t), p(t))=(x+2 t \xi, \xi)$ is the solution of $(1.3)$ and $\phi_{0}^{t}(x, \xi)$.

From Theorem 1, we can easily derive the classical limit of $T(h)$ applied to coherent states. For this purpose, it is natural to regard $x$ and $\xi$ as symmetric variables. Thus introduce $U_{h}$ by

$$
U_{h} f(x)=h^{-n / 4} f\left(h^{-1 / 2} x\right), \quad f \in L^{2}\left(R^{n}\right) .
$$

Put: $\bar{H}_{0}^{h}=U_{h}^{*} H_{0}^{h} U_{h}, \bar{H}^{h}=U_{h}^{*} H^{h} U_{n}$. Then the time-delay operator corresponding to $\left(\bar{H}_{0}^{h}, \bar{H}^{h}\right)$ is given by

$$
\bar{T}(h)=U_{h}^{*} T(h) U_{h} .
$$

Theorem 2. Under the assumptions of Theorem 1, let $\left(x_{0}, \xi_{0}\right) \in R^{2 n}$, with $\left|\xi_{0}\right|^{2} \in J$, and $f \in C_{0}^{\infty}(J)$ with $f\left(\left|\xi_{0}\right|^{2}\right)=1$. Denote

$$
W_{h}\left(x_{0}, \xi_{0}\right)=\exp \left(i h^{-1 / 2}\left(x \cdot \xi_{0}-x_{0} \cdot D_{x}\right)\right) \text {. }
$$

Then one has

$$
\lim _{h \rightarrow 0_{+}}\left\langle W_{h}\left(x_{0}, \xi_{0}\right)^{*} f\left(\bar{H}_{0}^{h}\right) \bar{T}(h) W_{h}\left(x_{0}, \xi_{0}\right) f, g\right\rangle=\tau \circ \Omega_{-}^{\mathrm{cl}}\left(x_{0}, \xi_{0}\right)\langle f, g\rangle
$$

for any $f, g \in L^{2}\left(\mathbf{R}^{n}\right)$.

Theorems 1 and 2 illustrate clearly the relation between quantum and classical time-delay. Remark that semiclassical approximation of scattering quantities was studied by several authors. See for example [18-20, 27, 28]. In particular, in [18], Robert and Tamura considered the asymptotic of trace of on-shell time-delay operator $T(\lambda, h) \in \mathscr{L}\left(L^{2}\left(S^{n-1}\right)\right)$ under the assumption $V(x)=O\left(|x|^{-n-\varepsilon_{0}}\right)$. In [27], I proved Theorems 1 and 2 for $V \in C_{0}^{\infty}\left(R^{n}\right)$. The method used there is based on the correspondence between quantum and classical dynamics $[21,22]$. By that method, the leading term of $T(h)$ in semiclassical approximation is easy to find. But serious difficulties arise in estimating remainders. In this paper, our proof for Theorems 1 and 2 relies heavily on 
energy decay estimates given in $\S 2$. These estimates enable us to obtain that there exists $T_{0}>0$ such that, with suitable microlocalization, one has:

$$
W_{ \pm}(h)=U(t, h)^{*} J^{ \pm} U_{0}(t, h)=O\left(h^{\infty}\right), \quad \text { in } \mathscr{L}\left(L^{2}\right)
$$

for $\pm t>T_{0}$. Here $J^{ \pm}$is outgoing (or incoming) $h$-parametrix constructed with a phase slightly different from that of Isozaki and Kitada [6]. Notice that the method of this paper can also be used to study the semiclassical approximation of wave operators. In this case the nontrapping condition is not needed, for the classical wave operators are well defined for $(x, \xi) \in R^{2 n}$ with $\xi \neq 0$. See [16].

The organization of this paper is as follows. In $\S 2$, we give microlocalized energy decay estimates for $U(t, h)$. The difference between Proposition 2.1 and my previous works $[25,26]$ is that under suitable microlocalizations, we can obtain estimates of the form: $O\left(h^{N}\langle t\rangle^{-N}\right)$, for $\pm t>0$ and for any $N>0$. These estimates are crucial to the semiclassical approximation of $T(h) . \S 3$ is devoted to classical dynamics. In $\S 4$, we give proof for Theorems 1 and 2. Finally we recall in the Appendix a semiclassical Egorov theorem, which is used in $\S 4$.

\section{Microlocalized TIME-DECAY ESTIMATES}

In this section, $V$ is assumed to be a long-range potential satisfying, for some $\varepsilon_{0}>0$,

$$
\left|\partial_{x}^{\alpha} V(x)\right| \leq C_{\alpha}\langle x\rangle^{-\varepsilon_{0}-|\alpha|}, \quad x \in \mathbf{R}^{n}, \alpha \in \mathbf{N}^{n}
$$

We want to give some microlocalized time-decay estimates on $U(t, h)$. In the case of fixed $h>0$, this type of question was considered by several authors (cf. [3, 5-9]). In the semiclassical case, the local geometrical form of the potential plays an important role. By method of incoming and outgoing parametrices of Isozaki and Kitada, I proved in $[25,26]$ that the nontrapping condition (1.4) is both necessary and sufficient to obtain optimal results of the form

$$
\left\|\langle x\rangle^{s} b_{ \pm}(x, h D) f\left(H^{h}\right) U(t, h)\langle x\rangle^{-s-r}\right\|=O\left(\langle t\rangle^{-r}\right), \quad \mp t>0,
$$

uniformly in $\left.h \in] 0, h_{0}\right]$, where $b_{ \pm} \in S_{ \pm}^{0}, f \in C_{0}^{\infty}(J)$ and $s, r \geq 0$. The following improvements are useful to semiclassical approximation of $T(h)$.

Proposition 2.1. Assume that $V$ satisfies (2.1). Let $J$ be an interval of nontrapping energy. Let $f \in C_{0}^{\infty}(J)$, and $m \in \mathbf{R}$. Then we have:

(i) For any $\sigma, d, R_{0}>0$, there exists $R=R\left(\sigma, d, R_{0}\right)>0$ such that for any $\chi \in C_{0}^{\infty}\left(B_{R_{0}}\right), b_{ \pm} \in S_{ \pm}^{m}(\sigma, d, R)$, one has

$$
\left\|\chi f\left(H^{h}\right) U(t, h) b_{ \pm}(\chi, h D)\right\| \leq C_{N} h^{N}\langle t\rangle^{-N}, \quad \pm t>0,0<h \leq h_{0},
$$

for any $N \in \mathbf{N}$. 
(ii) Assume that $b_{ \pm} \in S_{ \pm}^{m}\left(\sigma_{ \pm}, d_{ \pm}, R_{ \pm}\right)$with $\sigma_{+}+\sigma_{-}>2$ and $R_{+}+R_{-}$ large enough. Then for any $N \in \mathbf{N}$, one has

$$
\left\|b_{ \pm}(x, h D) f\left(H^{h}\right) U(t, h) b_{\mp}(x, h D)\right\| \leq C_{N} h^{N}\langle t\rangle^{-N}
$$

for $\left.\mp t>0, h \in] 0, h_{0}\right]$.

Proof. It can be easily checked that (2.2) and (2.3) are true if we replace $H^{h}$ by $H_{0}^{h}$ and $U(t, h)$ by $U_{0}(t, h)$. In the general case, we follow the line of [25, $26]$. By constructing outgoing and incoming $h$-parametrices as in [25], making use of Theorem 1 in [26], we can compare directly $U(t, h)$ with $U_{0}(t, h)$ and derive the desired results by the arguments already used in [25]. The details are omitted.

Corollary 2.2. Let $S_{ \pm}^{m}=\bigcup S_{ \pm}^{m}(\sigma, d, R)$, where the union is taken over $\sigma \in$ ]0, 2[ $, d>0, R>0$. For any $\chi_{1}, \chi_{2} \in C_{0}^{\infty}\left(\mathbf{R}_{x}^{n}\right), b_{ \pm} \in S_{ \pm}^{m}$, there exists some $T_{0}>0$ such that

$$
\begin{gathered}
\left\|\chi_{1} f\left(H^{h}\right) U(t, h) \chi_{2}\right\|=O\left(h^{\infty}\langle t\rangle^{-\infty}\right), \quad|t|>T_{0}, \\
\left\|\chi_{1} f\left(H^{h}\right) U(t, h) b_{ \pm}(x, h D)\right\|=O\left(h^{\infty}\langle t\rangle^{-\infty}\right), \quad \pm t>T_{0} .
\end{gathered}
$$

If $B_{ \pm} \in S_{ \pm}^{m}\left(\sigma_{ \pm}, d_{ \pm}, R_{ \pm}\right)$with $\sigma_{+}+\sigma_{-}>2$, then there exists $T_{0}>0$ such that

$$
\left\|b_{\mp}(x, h D) f\left(H^{h}\right) U(t, h) b_{ \pm}(x, h D)\right\|=O\left(h^{\infty}\langle t\rangle^{-\infty}\right), \quad \pm t>T_{0} .
$$

Here $f \in C_{0}^{\infty}(J)$ and $J$ is an interval of nontrapping energy.

Proof. Take $g \in C_{0}^{\infty}(J)$ such that $g=1$ on supp $f$. Then we can write

$$
g\left(H^{h}\right) \chi_{2}(x)=b(x, h D ; h)+R(h)
$$

where $\left\|\langle x\rangle^{N} R(h)\langle x\rangle^{N}\right\|=O\left(h^{N}\right)$ for any $N>0$ and $b(h)$ is a symbol with support in $\left\{(x, \xi) ; x \in \operatorname{supp} \chi_{2}, p(x, \xi) \in \operatorname{supp} g\right\}$. By a semiclassical Egorov theorem (see the Appendix), one has

$$
U(T, h) b(x, h D ; h)=b_{T}(x, h D ; h) U(T, h)+R_{T}(h), \quad T>0,
$$

where $R_{T}(h)$ has the same properties as $R(h)$ and

$$
\operatorname{supp} b_{T}(h) \subset \operatorname{supp} b\left(\phi^{-T}, h\right) \text {. }
$$

Here $\phi^{t}$ is the Hamilton flow for $p=|\xi|^{2}+V(x)$. Therefore if $(x, \xi) \in$ supp $b_{T}(h)$, we can write: $(x, \xi)=\phi^{T}(y, \eta)$ for some $(y, \eta) \in \operatorname{supp} b(h)$. Since $|y| \leq R_{0}$ for some $R_{0}>0$ independent of $T$, the nontrapping assumption implies that for any $R>0$ there exists $T_{0}>0$ such that for $T>T_{0}$, $\operatorname{supp} b_{T}(h) \subset \Omega_{+}\left(1, d_{0}, R\right)$. If $R>0$ is large enough, we can apply (2.2) to obtain that

$$
\left\|\chi_{1} f\left(H^{h}\right) U(t, h) b(x, h D ; h)\right\|=O\left(h^{\infty}\langle t\rangle^{-\infty}\right), \quad t>T_{0} .
$$


From Theorem 1 in [26] and (2.8), one derives (2.4) for $t>T_{0}$. The case $-t>T_{0}$ can be obtained by taking the adjoint. Equations (2.5) and (2.6) can be proved in the same way. It should be noticed that if $I$ is a compact subinterval contained in $J$, then there exist $C>0$ and $t_{0}>0$ such that $|q(t ; x, \xi)| \geq C(|x|+t|\xi|)$, for $t>t_{0},(x, \xi) \in \operatorname{supp} b_{+} \cap p^{-1}(I)$. In particular, one can derive that for any $\varepsilon>0$ and $R>0$, one can take $T>0$ large enough such that

$$
\phi^{T}\left(\operatorname{supp} b_{+} \cap p^{-1}(I)\right) \subset \Omega_{+}(2-\varepsilon, d, R) .
$$

This enables us to obtain (2.5) and (2.6) from Proposition 2.1. The details are omitted.

\section{Classical dynamics}

From now on, the potential $V$ is always assumed to satisfy (1.1). In this section, we want to establish some results on classical dynamics, which are useful in the next section. Our first result is concerned with an improvement of a result of Isozaki and Kitada [6, Proposition 2.4]. This improvement is of course due to the short range assumption on $V$, but it does not follow directly from the construction given in [6].

Lemma 3.1. Let $V$ satisfy (1.1). Then for any $\varepsilon, d>0$, there exist two real functions $\phi_{ \pm} \in C^{\infty}\left(\mathbf{R}^{2 n}\right)$ such that for $R$ large enough, $\phi_{ \pm}$satisfies the eikonal equation

$$
\left|\nabla_{x} \phi_{ \pm}(x, \xi)\right|^{2}+V(x)=|\xi|^{2} \quad \text { in } \Omega_{ \pm}\left(\varepsilon, d, R_{0}^{*}\right)
$$

and for any multi-indices $\alpha, \beta \in \mathbf{N}^{n}$,

$$
\left|\partial_{x}^{\alpha} \partial_{\xi}^{\beta}\left(\phi_{ \pm}(x, \xi)-x, \xi\right)\right| \leq C_{\alpha \beta}\langle x\rangle^{-\varepsilon_{0}-|\alpha|}, \quad(x, \xi) \in R^{2 n} .
$$

Proof. Let $\phi^{t}(x, \xi)=(q(t ; x, \xi), p(t ; x, \xi))$ denote the solution of Hamilton system (1.3). Then we can verify that for $0<\varepsilon<2 d>0$, there exist some $C>0$ and $R_{0}>0$ such that for $R>R_{0},(x, \xi) \in \Omega_{+}(\varepsilon, d, R)$, we have

$$
\phi^{t}(x, \xi) \in \Omega_{+}(\varepsilon / 2, d / 2, R / C) \text { and }|q(t ; x, \xi)| \geq C^{-1}(|x|+t|\xi|)
$$

for all $t>0$. Using the expression

$$
p(t ; x, \xi)=\xi-\int_{0}^{t} \nabla V(q(s ; x, \xi)) d s,
$$

we can easily derive that

$$
|p(t ; x, \xi)-\xi| \leq C\langle x\rangle^{-1-\varepsilon_{0}}, \quad \text { for all } t>0 .
$$

For $|x|>R$, define $\Gamma_{+}(\varepsilon, d)=\left\{\xi \in R^{n} ;(x, \xi) \in \Omega_{+}(\varepsilon, d, R)\right\}$. From the above estimates, it follows that for $R>0$ large enough, and for fixed $x$ with $|x|>R$, the map $\xi \rightarrow p(t ; x, \xi)$ is a global diffeomorphism from $\Gamma_{+}(\varepsilon, d)$ onto its image, whose union, when $x$ ranges over the domain $\{|y|>R\}$, 
contains a set of the form $\Omega_{+}(2 \varepsilon, 2 d, C \mathbf{R})$ for all $t>0$. Let $\xi \rightarrow \eta(t ; x, \xi)$ be the inverse to $\xi \rightarrow p(t ; x, \xi)$. Put

$$
y(t ; x, \xi)=q(t ; x, \eta(t ; x, \xi)) \text {. }
$$

Then one has:

$$
\begin{aligned}
y(t ; x, \xi) & =x+2 t \xi+\int_{0}^{t} 2 s \nabla V(q(s ; x, \eta(t ; x, \xi)) d s \\
& =x+2 t \xi+O\left(\langle x\rangle^{-\varepsilon_{0}}\right) .
\end{aligned}
$$

From this estimate, one derives easily that $\nabla_{x} y(t ; x, \xi)=I+O\left(\langle x\rangle^{-\varepsilon_{0}}\right)$. With a little more work, we can in fact prove that

$$
\nabla_{x} y(t ; x, \xi)=I+O\left(\langle x\rangle^{-1-\varepsilon_{0}}\right)
$$

uniformly in $t>0$. Finally we define as in [6]

$$
\begin{aligned}
& u(t ; x, \xi)=x \cdot \xi+\int_{0}^{t}(p-x \cdot \nabla V)\left(\phi^{s}(x, \xi)\right) d s \\
& \phi(t ; x, \xi)=u(t ; x, \eta(t ; x, \xi))
\end{aligned}
$$

for $(x, \xi) \in \Omega_{+}\left(\varepsilon, d, R_{0}\right)$, with $R_{0}=R_{0}(\varepsilon, d)$ large enough. Then by the Hamilton-Jacobi theory,

$$
\left\{\begin{array}{l}
\frac{\partial}{\partial t} \phi(t ; x, \xi)=|\xi|^{2}+V\left(\nabla_{\xi} \phi(t ; x, \xi)\right)=\left|\nabla_{x} \phi(t ; x, \xi)\right|^{2}+V(x) \\
\phi(0 ; x, \xi)=x \cdot \xi \text { for }(x, \xi) \in \Omega_{+}\left(\varepsilon, d, R_{0}\right)
\end{array}\right.
$$

and

$$
\partial_{x} \phi(t ; x, \xi)=\eta(t ; x, \xi) ; \quad \partial_{\xi} \phi(t ; x, \xi)=y(t ; x, \xi)
$$

Now instead of taking $\phi_{+}$as the $\operatorname{limit} \lim _{t \rightarrow+\infty}(\phi(t ; x, \xi)-\phi(t ; 0, \xi))$, we define it as

$$
\phi_{+}(x, \xi)=\lim _{t \rightarrow+\infty}\left(\phi(t ; x, \xi)-t|\xi|^{2}\right) \text {. }
$$

Then $\phi_{+}$satisfies the eikonal equation in $\Omega_{+}\left(\varepsilon, d, R_{0}\right)$ and

$$
\phi_{+}(x, \xi)-x \cdot \xi=\int_{0}^{+\infty} V(y(t ; x, \xi)) d t, \quad(x, \xi) \in \Omega_{+}\left(\varepsilon, d, R_{0}\right) .
$$

From (3.4) and similar estimates over higher derivatives, it follows that (3.2) is satisfied. Now to finish the proof of lemma for $\phi_{+}$, it suffices to introduce a suitable cut-off function to make $\phi_{+}$globally defined on $\mathbf{R}^{2 n} \cdot \phi_{-}$can be constructed in a similar way.

Take $\chi \in C_{0}^{\infty}\left(\mathbf{R}^{n}\right), \chi(x)=0$ for $|x| \leq 1, \chi(x)=1$ for $|x| \geq 2$. For $R_{1}>R_{0}$, replacing $\phi_{ \pm}$by $\left(\phi_{ \pm}(x, \xi)-x \cdot \xi\right) \chi\left(x / R_{1}\right)+x \cdot \xi$ in $(3.1)$, one sees clearly that we can assume without loss that $\phi_{ \pm}$satisfies:

$$
\begin{gathered}
\left|\nabla_{x} \phi_{ \pm}(x, \xi)\right|^{2}+V(x)=|\xi|^{2} \text { in } \Omega_{ \pm}\left(\varepsilon, d, R_{1}\right), \\
\left|\partial_{x}^{\alpha} \partial_{\xi}^{\beta}\left(\phi_{ \pm}(x, \xi)-x \cdot \xi\right)\right| \leq c_{\alpha \beta} R_{1}^{-\varepsilon_{1}}\langle x\rangle^{-|\alpha|-\varepsilon_{2}}, \quad(x, \xi) \in \mathbf{R}^{2 n},
\end{gathered}
$$


with $\varepsilon_{1}+\varepsilon_{2}=\varepsilon_{0}, \varepsilon_{j} \geq 0$, and $C_{\alpha \beta}$ independent of $R_{1}$. For $R_{1}$ large enough, the maps $\xi \rightarrow \nabla_{x} \phi_{ \pm}(x, \xi), x \rightarrow \nabla_{\xi} \phi_{ \pm}(x, \xi)$ are global diffeomorphisms. Let $\eta_{ \pm}(x, \xi), y_{ \pm}(x, \xi)$ denote the inverse diffeomorphism:

$$
\nabla_{x} \phi_{ \pm}\left(x, \eta_{ \pm}(x, \xi)\right)=\xi, \quad \nabla_{\xi} \varphi_{ \pm}\left(y_{ \pm}(x, \xi), \xi\right)=x, \quad(x, \xi) \in R^{2 n}
$$

Lemma 3.2. Let $(q(t ; x, \xi), p(t ; x, \xi))$ denote the solution of the Hamilton system (1.3). Then for any $\sigma, d>0$, there exists $R_{1}>0$ such that for $(x, \xi) \in$ $\Omega_{ \pm}\left(\sigma, d, R_{1}\right)$, one has

(3.10) $q(t ; x, \xi)=\nabla_{\xi} \phi_{ \pm}\left(x, \eta_{ \pm}(x, \xi)\right)+2 t \eta_{ \pm}(x, \xi)+O\left(\langle t\rangle^{-\varepsilon_{0}}\right), \quad t \rightarrow \pm \infty$.

In particular for $(x, \xi) \in \Omega_{ \pm}\left(\sigma, d, R_{1}\right)$,

$$
\left\{\begin{array}{l}
\nabla_{\xi} \phi_{ \pm}\left(x, \eta_{ \pm}(x, \xi)\right)=x+\int_{0}^{ \pm \infty} s \nabla V(q(s ; x, \xi)) d s, \\
\eta_{ \pm}(x, \xi)=\xi-\int_{0}^{ \pm \infty} \nabla V(q(s ; x, \xi)) d s .
\end{array}\right.
$$

Proof. Consider only the case $t \rightarrow+\infty$. Recall that for $(x, \xi) \in \Omega_{+}\left(\sigma, d, R_{1}\right)$ with $R_{1}$ large enough, one has

$$
\phi_{+}(x, \xi)=\lim _{t \rightarrow+\infty}\left(\phi(t ; x, \xi)-t \xi^{2}\right)
$$

where $\phi(t ; x, \xi)$ satisfies (3.5) and (3.6). It is easy to check that

$$
\partial_{\xi} \phi(t ; x, \xi)=\nabla_{\xi} \phi_{+}(x, \xi)+2 t \xi+o(1), \quad t \rightarrow+\infty .
$$

Since for $(x, \xi) \in \Omega_{+}\left(\sigma, d, R_{1}\right)$, the limit $\eta(\infty, x, \xi)=\lim _{t \rightarrow+\infty} \eta(t ; x, \xi)$ exists and

$$
\begin{gathered}
\left|\partial_{x}^{\alpha} \partial_{\xi}^{\beta} q(t ; x, \xi)\right| \leq C_{\alpha \beta}\langle t\rangle, \\
\left|\partial_{x}^{\alpha} \partial_{\xi}^{\beta}(\eta(t ; x, \xi)-\eta(\infty, x, \xi))\right| \leq C_{\alpha \beta}\langle t\rangle^{-1-\varepsilon_{0}},
\end{gathered}
$$

one derives from the equality $\partial_{\xi} \phi(t ; x, \xi)=q(t ; x, \eta(t ; x, \xi))$ that

$$
q(t ; x, \eta(\infty, x, \xi))=\partial_{\xi} \phi_{+}(x, \xi)+2 t \xi+o(1), \quad t \rightarrow+\infty .
$$

Noticing that $\eta(\infty ; x, \xi)=\lim _{t \rightarrow \infty} \nabla_{x} \phi_{+}(t ; x, \xi)=\nabla_{x} \phi_{+}(x, \xi)$, (3.10) for $t \rightarrow+\infty$ follows easily from (3.12) by returning to the Hamilton system.

Remark that for the phase functions constructed by Isozaki and Kitada, one has

$$
q(t ; x, \xi)=a_{ \pm}(x, \xi)+2 t \eta_{ \pm}+o\left(\langle t\rangle^{-\varepsilon_{0}}\right), \quad t \rightarrow \pm \infty,
$$

where $a_{ \pm}(x, \xi)=\nabla_{\xi} \phi_{ \pm}\left(x, \eta_{ \pm}(x, \xi)\right)+b_{ \pm}(\xi), b_{ \pm} \neq 0$. This is clear from their definition of $\phi_{ \pm}$and from an estimate similar to (3.2). Now let $\sigma, d$, and $R_{1}$ be as in Lemma 3.2 and $\phi_{ \pm}$be solutions to the eikonal equation constructed in Lemma 3.1. Let $a_{0, \pm}$ denote the solution to the equation

$$
2 \nabla_{x} \phi_{ \pm} \cdot \nabla_{x} a_{0, \pm}+\Delta_{x} \phi_{ \pm} a_{0, \pm}=0, \quad \text { in } \Omega_{ \pm}\left(2 \sigma, 2 d, 2 R_{1}\right),
$$

with the condition $a_{0, \pm}(x, \xi)=1+o\left(\langle x\rangle^{-\varepsilon_{0}}\right),(x, \xi) \in \Omega_{ \pm}\left(2 \sigma, 2 d, 2 R_{1}\right)$. Such solutions exist [25] and are in fact the principal symbol of the outgoing and incoming $h$-parametrices used in $\S 4$. 
Lemma 3.3. Let $a_{0, \pm}$ be defined as above. Then for $(x, \xi) \in \Omega_{ \pm}\left(2 \sigma, 2 d, 2 R_{1}\right)$, one has

$$
a_{0, \pm}(x, \xi)=\left|\operatorname{det}\left(\partial_{x} \partial_{\xi} \phi_{ \pm}(x, \xi)\right)\right|^{1 / 2} .
$$

Proof. Consider only $a_{0, \pm}$. As in [25], we can write

$$
a_{0,+}(x, \xi)=e^{\frac{1}{2} \int_{0}^{+\infty} \Delta_{x} \phi_{+}(\rho(t ; x, \xi), \xi) d t}
$$

where $\rho(t)$ is the solution of the problem

$$
\dot{\rho}(t)=\nabla_{x} \phi_{+}(\rho(t), \xi), \quad \rho(0 ; x, \xi)=x .
$$

By the proof of Lemma 3.2, $q_{0}(t ; x, \xi) \equiv q\left(t ; x, \nabla_{x} \phi_{+}(x, \xi)\right)$ satisfies

$$
\left\{\begin{array}{l}
q_{0}(0 ; x, \xi)=x, \quad \lim _{t \rightarrow+\infty} q_{0}(t ; x, \xi)=2 \xi, \\
\partial_{x} q_{0}(t ; x, \xi)=\partial_{x} \partial_{\xi} \phi_{+}(x, \xi)+O\left(\langle t\rangle^{-\varepsilon_{0}}\right), \quad t \rightarrow+\infty .
\end{array}\right.
$$

In addition, by the Hamilton-Jacobi theory, we have:

$$
\dot{q}_{0}(t ; x, \xi)=\nabla_{x} \phi_{+}\left(q_{0}(t ; x, \xi), \xi\right) .
$$

This means: $\rho(t ; x, \xi)=q_{0}(t ; x, \xi)$ for $t>0$ and $(x, \xi) \in \Omega_{+}\left(2 \sigma, 2 d, 2 R_{1}\right)$ with $R_{1}>0$ large enough. From Liouville theorem (see [12]), we obtain

$$
\exp \left(\int_{0}^{t} \Delta_{x} \phi_{+}\left(q_{0}(s ; x, \xi), \xi\right) d s\right)=\left|\operatorname{det}\left(\partial_{x} q_{0}(t ; x, \xi)\right)\right| \text {. }
$$

Utilizing (3.14) and taking the limit $t \rightarrow+\infty$, we obtain Lemma 3.3.

\section{Semiclassical approximation of time-delay}

Our study of time-delay operator $T(h)$ is based on (1.2). Let $J$ be an interval of nontrapping energy. For $f \in C_{0}^{\infty}(J)$, one has

$$
f\left(H_{0}\right) T(h)=f_{1}\left(H_{0}^{h}\right) S(h)^{*}[S(h), A(h)]
$$

where $f_{1}(t)=f(t) / 2 t, t>0$. Therefore, the semiclassical approximation of $T(h)$ is closely related to that for $S(h)$. However, as already remarked in [27], although $S(h)$ can only be approximated by Fourier integral operators, $T(h)$ can be well approximated by $h-\psi$ DOs. We begin with constructing approximation for wave operators.

For $d>0$, take $\chi \in C^{\infty}(\mathbf{R})$ such that $\chi(\lambda)=0$ for $\lambda<d / 3$ and $\chi(\lambda)=1$ for $\lambda>d / 2$. For $\varepsilon>0$ small enough, choose $\rho \in C^{\infty}(\mathbf{R})$ such that $\rho(r)=0$ for $r<-\varepsilon$ and $\rho(r)=1$ for $r \geq 0$. Let $\theta \in C_{0}^{\infty}\left(\mathbf{R}^{n}\right), \theta(x)=1$ for $|x| \leq 1$. Then we have for any $R>0$,

$$
\chi\left(H_{0}^{h}\right)=\theta\left(\frac{x}{R}\right) \chi\left(H_{0}^{h}\right)+\widehat{b}_{-}(x, h D)+b_{+}(x, h D)
$$

where

$$
\begin{gathered}
\tilde{b}_{-}(x, \xi)=\left(1-\theta\left(\frac{x}{R}\right)\right) \chi\left(|\xi|^{2}\right)(1-\rho(\widehat{x} \cdot \widehat{\xi}-1+\varepsilon), \\
b_{+}(x, \xi)=\left(1-\theta\left(\frac{x}{R}\right)\right) \chi\left(|\xi|^{2}\right) \rho(\widehat{x}, \widehat{\xi}-1+\varepsilon) .
\end{gathered}
$$


Denote $E_{d}(h)$ the spectral projector of $H_{0}^{h}$ onto the interval $[d,+\infty[$. From the estimates

$$
\begin{gathered}
\left\|\theta\left(\frac{x}{R}\right) \chi\left(H_{0}^{h}\right) U_{0}(t, h)\langle x\rangle^{-1}\right\| \leq C\langle t\rangle^{-1}, \quad t \in R, \\
\left\|\widetilde{b}_{-}(x, h D) U_{0}(t, h)\langle x\rangle^{-1}\right\| \leq C\langle t\rangle^{-1}, \quad t>0,
\end{gathered}
$$

we derive easily that for $f \in \operatorname{Ran} E_{d}(h)$,

$$
\begin{gathered}
W_{+}(h) f=\lim _{t \rightarrow+\infty} U(t, h)^{*} U_{0}(t, h) f \\
=\lim _{t \rightarrow+\infty} U(t, h)^{*} b_{+}(x, h D) U_{0}(t, h) f \quad \text { in } L^{2}\left(R^{n}\right) . \\
W_{+}(h)=\underset{t \rightarrow+\infty}{s-\lim _{t \rightarrow+\infty}}\left\{U(t, h)^{*} J^{+}\left(a_{+}(h)\right) U_{0}(t, h)\left[U_{0}(t, h)^{*} J^{+}(b)^{*} U_{0}(t, h)\right]\right\}
\end{gathered}
$$

on $\operatorname{Ran} E_{d}(h)$.

Lemma 4.1. The limit $\mathrm{s}-\lim _{t \rightarrow+\infty} U_{0}(t, h)^{*} J^{+}(b)^{*} U_{0}(t, h)$ exists in $L^{2}\left(R^{n}\right)$ and is equal to $I$ on the range of $E_{d}(h)$ :

$$
\lim _{t \rightarrow+\infty} U_{0}(t, h)^{*} J^{+}(b)^{*} U_{0}(t, h) f=f, \quad \forall f \in \operatorname{Ran} E_{d}(h) .
$$

Proof. We give only the proof of (4.3). Notice that

$$
J^{+}(b)^{*} u(x)=(2 \pi h)^{-n} \iint_{\mathbf{R}^{2 n}} e^{i(x-y) \cdot \xi / h} C(y, \xi ; h) u(y) d y d \xi,
$$

where $C(y, \xi ; h)=e^{i\left(y \cdot \xi-\varphi_{+}(y, \xi)\right) / h} \bar{b}(y, \xi)$. For fixed $h>0, C(h) \in S^{0}$. Therefore, if $C^{W}(x ; h D ; h)$ denotes the $h-\psi$ DO with Weyl symbol $C(h)$, then

$$
J^{+}(b)^{*}=C^{W}(x, h D ; h)+r_{1}(x, h D ; h)
$$

where $r_{1}(h) \in S^{-1}$. An easy calculus gives

$$
\underset{t \rightarrow+\infty}{\mathrm{s}-\lim _{0}} U_{0}(t, h)^{*} J^{+}(b)^{*} U_{0}(t, h)=\underset{t \rightarrow+\infty}{\mathrm{s}-\lim _{0}} U_{0}(t, h)^{*} C^{W}(x, h D) U_{0}(t, h) .
$$

$U_{0}(t, h)^{*} C^{W}(x, h D ; h) U_{0}(t, h)$ is an $h-\psi$ DO with the Weyl symbol $C(x+2 t \xi, \xi ; h)$. As $t \rightarrow+\infty, b(x+2 t \xi, \xi) \rightarrow 1$ for $|\xi|^{2} \geq d$ and by Lemma 3.1:

$$
\left|(x+2 t \xi) \cdot \xi-\phi_{+}(x+2 t \xi ; \xi)\right| \leq C\langle x+2 t \xi\rangle^{-\varepsilon_{0}} \rightarrow 0, \quad|\xi|^{2} \geq d .
$$

Therefore, $C^{W}(x+2 t h D, h D ; h) f \rightarrow f$ for any $f \in \operatorname{Ran} E_{d}(h)$. This proves (4.3).

According to Lemma 4.1, for $f \in \operatorname{Ran} E_{d}(h),(4.2)$ is true for any $\varepsilon>0$, $R>0$. Similarly,

$$
W_{-}(h) f=\lim _{t \rightarrow-\infty} U(t, h)^{*} b_{-}(x, h D) U_{0}(t) f
$$

where $b_{-}(x, \xi)=\left(1-\theta\left(\frac{x}{R}\right)\right) \chi\left(|\xi|^{2}\right) p(-1+\varepsilon-\widehat{x} \cdot \widehat{\xi})$. We want to prove that for some parametrices $J^{ \pm}\left(a_{ \pm}(h)\right)$,

$$
W_{ \pm}(h)=s-\lim _{t \rightarrow \pm \infty} U(t, h)^{*} J^{ \pm}\left(a_{ \pm}(h)\right) U_{0}(t, h) \quad \text { on } \operatorname{Ran} E_{d}(h) .
$$


In fact $J^{ \pm}$is just an outgoing (incoming parametrix constructed as in [25]. However as remarked in [10], for phase functions given in [6], one cannot have (4.5). But we shall see that with the modification on the phase functions given in Lemma $3.1,(4.5)$ is really true.

Let $\varepsilon>0, d>0$ be sufficiently small and $\phi_{ \pm}$solutions to (3.7). For a symbol $a$, denote by $J^{ \pm}(a)$ the Fourier integral operators defined by:

$$
J^{ \pm}(a) u(x)=(2 \pi h)^{-n} \iint_{\mathbf{R}^{2 n}} e^{i\left(\varphi_{ \pm}(x, \xi)-y \cdot \xi\right) / h} a(x, \xi) u(y) d y d \xi
$$

for $u \in \mathscr{S}\left(R^{n}\right)$. Let $a_{ \pm}(h)$ denote the $h$-parametrices constructed from $\phi_{ \pm}$ given above, by a procedure completely similar to that described in [25] (see also [6]), such that if we define

$$
r_{ \pm}(h)=e^{-i \varphi_{ \pm} / h}\left(H^{h}-|\xi|^{2}\right)\left(e^{i \varphi_{ \pm} / h} a_{ \pm}(h)\right),
$$

then $r_{ \pm}(h) \in S_{ \pm}^{-1,1}\left(\varepsilon, d, R_{1}\right)$ and

$$
r_{ \pm}(x, \xi ; h)=O\left(h^{\infty}\langle x\rangle^{-\infty}\right), \quad \text { for }(x, \xi) \in \Omega_{ \pm}\left(2 \varepsilon, 2 d, 2 R_{1}\right) .
$$

For $b_{+}$defined in (4.2) with $R \gg R_{1}$, we can find $b \in S_{+}^{0}$ such that

$$
\left\{\begin{array}{l}
b(x, \xi)=1+O\left(\langle x\rangle^{-1}\right) \text { in } \Omega_{+}\left(2-\frac{\varepsilon}{2}, d, 4 R\right), \\
J^{+}\left(a_{+}(h)\right) J^{+}(b)^{*}=b_{+}(x, h D) \neq r_{0}(x, h D ; h)
\end{array}\right.
$$

with $r_{0}(h) \in S_{+}^{-1,1}$. From (4.2) we have

$$
W_{+}(h) f=\lim _{t \rightarrow+\infty} U(t, h)^{*} J^{+}\left(a_{+}(h)\right) U_{0}(t, h) f \quad \text { in } L^{2}\left(\mathbf{R}^{n}\right)
$$

or formally,

$$
W_{+}(h)=J^{+}\left(a_{+}(h)\right)+i h^{-1} \int_{0}^{+\infty} U(t, h)^{*} J^{+}\left(r_{+}(h)\right) U_{0}(t, h) d t
$$

on $\operatorname{Ran} E_{d}(h)$. (4.10) shows that $J^{+}\left(a_{+}(h)\right)$ is a good approximation for $W_{+}(h)$. Similarly, we can show that $J^{-}\left(a_{-}(h)\right)$ is a good approximation for $W_{-}(h)$. Put

$$
W_{ \pm}(t, h)=U(t, h)^{*} J^{ \pm}\left(a_{ \pm}(h)\right) U_{0}(t, h) .
$$

Then by the completeness of wave operators, one has

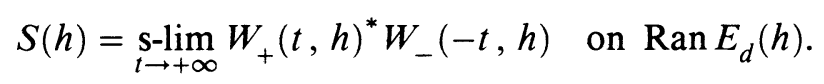

Lemma 4.2. Let $J$ be an interval of nontrapping energy, $\chi \in C_{0}^{\infty}(J), \phi \in$ $C_{0}^{\infty}\left(R^{+}\right)$. Then for any $s \in[-1,1]$, one has

$$
\left\|\langle A\rangle^{s} \chi\left(H^{h}\right) W_{ \pm}(t, h) f\left(H_{0}^{h}\right)\langle A\rangle^{-s}\right\| \leq C
$$


uniformly in $\left.t \in T, h \in] 0, h_{0}\right]$. Here $A=h\left(x \cdot D_{x}+D_{x} \cdot x\right) / 2,\langle A\rangle=$ $\left(1+|A|^{2}\right)^{1 / 2}$.

Proof. Consider only $W_{+}(t, h)$. By the construction of $J^{+}\left(a_{+}(h)\right)$, one has

$$
W_{+}(t, h)=J^{+}\left(a_{+}(h)\right)+i h^{-1} \int_{0}^{t} U(-\tau, h) J^{+} U_{0}(\tau, h) d \tau
$$

where $J^{+}=J^{+}\left(R_{+}(h)\right)$. Clearly it suffices to discuss the commutator of the second term in the right-hand side of (4.13) with $A$. Take $\chi_{1}, \chi_{2} \in C_{0}^{\alpha}(J)$ with $\chi_{1}=1$ on $\operatorname{supp} \chi$ and $\chi_{2}=1$ on $\operatorname{supp} \chi_{1}$. We can write

$$
\begin{aligned}
{[A, \chi(} & \left.\left.H^{h}\right) U(\tau, h)^{*} J^{+} U_{0}(\tau, h) f\left(H_{0}^{h}\right)\right] \\
= & {\left[A, \chi\left(H^{h}\right)\right] U(\tau, h)^{*} \chi_{1}\left(H^{h}\right) J^{+} U_{0}(\tau, h) f\left(H_{0}^{h}\right) } \\
& +\chi\left(H^{h}\right) U(\tau, h)^{*}\left[A, J^{+}\right] U_{0}(\tau, h) f\left(H_{0}^{h}\right) \\
& +\chi\left(H^{h}\right) U(\tau, h)\left[A, \chi_{1}\left(H^{h}\right)\right] J^{+} U_{0}(\tau, h) f\left(H_{0}^{h}\right) \\
& +\chi\left(H^{h}\right) U(\tau, h)^{*} J^{+} U_{0}(\tau, h)\left[A, f\left(H_{0}^{h}\right)\right] \\
& +\int_{0}^{\tau} \chi\left(H^{h}\right) U(s, h)^{*} \widetilde{V} U(s-\tau, h) d s \chi_{1}\left(H^{h}\right) J^{+} U_{0}(\tau, h) f\left(H_{0}^{h}\right) \\
& +2 \tau \chi\left(H^{h}\right) U(\tau, h)^{*}\left(J^{+} H_{0}^{h}-H^{h} J^{+}\right) U_{0}(\tau, h) f\left(H_{0}^{h}\right) \\
\equiv & \sum_{j=1}^{6} B_{j}(\tau, h) .
\end{aligned}
$$

Here we used the commutator relation

$$
[A, U(t, h)]=2 t H^{h} U(t, h)+\int_{0}^{t} U(t-s, h) \widetilde{V} U(s) d s
$$

with $\widetilde{V}=x \cdot \nabla_{x} V-2 V$. To estimate the $B_{j}(t, h)$ we need the following two estimates:

$$
\left\|\int_{0}^{\tau} \chi\left(H^{h}\right) U(s, H)^{*} \tilde{V} U(s ; h) \chi_{1}\left(H^{h}\right) d s\right\| \leq C
$$

uniformly in $\tau \in \mathbf{R}, h \in] 0, h_{0}$ ], and for any $\varphi \in C_{0}^{\infty}(J), b_{1} \in S_{+}^{-1 / 2} \cap S_{-}^{-1 / 2}$,

$$
\left|\int_{0}^{t}\left\|b_{1}(x, h D) U(s, h) \varphi\left(H^{h}\right) g\right\|^{2} d s\right| \leq C\|g\|^{2}, \quad g \in L^{2}\left(\mathbf{R}^{n}\right)
$$

uniformly in $t \in \mathbf{R}$ in $h \in$ ]0, $h_{0}$ ]. (4.14) was proved in [27]. To prove (4.15) recall that I proved in [25] that for $b_{ \pm} \in S_{ \pm}^{0}, S>\frac{1}{2}$,

$$
\left\|\langle x\rangle^{-s} R(\lambda \pm i 0, h) b_{ \pm}(x, h D)\langle x\rangle^{s-1}\right\| \leq C h^{-1}, \quad \lambda \in J .
$$

Therefore if $b \in S_{+}^{0} \cap S_{-}^{0}$, and $s=0,1$, one has

$$
\left\|\langle x\rangle^{-s} b(x, h D) R(\lambda \pm i 0 ; h) b(x, h D)\langle x\rangle^{s-1}\right\| \leq C h^{-1} .
$$


By an easy interpolation, one obtains

$$
\left\|\langle x\rangle^{-1 / 2} b(x, h D) R(\lambda \pm i 0, h) b(x, h D)\langle x\rangle^{-1 / 2}\right\| \leq C h^{-1} .
$$

From (4.16), one can derive (4.15) by the local $H^{h}$-smoothness of $b_{1}(x, h D)$. See also the proof of Proposition 2.3 in [27]. Since $r_{+}(h) \in S_{+}^{-1,1}\left(\varepsilon, d, R_{1}\right)$ and $r_{+}(x, \xi ; h)=O\left(h^{\infty}\langle x\rangle^{-\infty}\right)$ in $\Omega_{+}\left(2 \varepsilon, 2 d, 2 R_{1}\right)$, we can write

$$
J^{+}=h\left\{b_{1}(x, h D) J^{+}\left(r_{1}(h)\right) b_{2}(x, h D)+J^{+}\left(r_{2}(h)\right)\right\}
$$

where $b_{j} \in S_{+}^{-1 / 2} \cap S_{-}^{-1 / 2}, r_{1}(h) \in S^{0,0}$ and $r_{2}(h) \in S^{-\infty, 0}$. From (4.15) and a similar result for $U_{0}(t, h)$, we derive easily that

$$
\left\|\int_{0}^{t} U(\tau, h)^{*} \varphi\left(H^{h}\right) J^{+} U_{0}(\tau, h) f\left(H_{0}^{h}\right) d \tau\right\| \leq C h
$$

uniformly in $t \in \mathbf{R}$ and $\left.h \in] 0, h_{0}\right]$. Since $\left[A, J^{+}\right]$has similar properties as $J^{+}$, it follows from (4.17) that

$$
\left\|\int_{0}^{t} h^{-1} B_{j}(\tau, h) d \tau\right\| \leq C, \quad j=1,2,
$$

uniformly in $t$ and $h$. By the relations (see [27])

$$
\begin{aligned}
& i\left[A, \chi_{2}\left(H^{h}\right)\right]=h\left(2 H^{h} \chi_{2}^{\prime}\left(H^{h}\right)+R_{1}(h),\right. \\
& i\left[A, f\left(H_{0}^{h}\right)\right]=h 2 H_{0}^{h} f^{\prime}\left(H_{0}^{h}\right),
\end{aligned}
$$

where $R_{1}(h)$ is an $h$-admissible operator in the sense of [4] with order $-1-\varepsilon_{0}$ in $x$, we can easily prove that (4.18) is also true for $j=3,4$. For $j=5$, one has

$$
\begin{aligned}
\left\|\int_{0}^{t} B_{5}(\tau, h) d \tau\right\| \leq & \left(\operatorname{supp}_{\tau}\left\|\int_{0}^{\tau} \chi\left(H^{h}\right) U(s, h)^{*} \widetilde{V} \chi_{1}\left(H^{h}\right) U(s, h) d s\right\|\right) \\
& \times\left\|\int_{0}^{t} \chi_{2}\left(H^{h}\right) U(\tau, h)^{*} J^{+} U_{0}(\tau, h) f\left(H_{0}^{h}\right) d \tau\right\| \\
\leq & C h
\end{aligned}
$$

uniformly in $t$. Here one used (4.14) and (4.17). Finally for $j=6$, we notice that $\phi_{+}$solves the eikonal equation on $\operatorname{supp} r_{+}(h)$. Hence,

$$
J^{+} H_{0}^{h}-H^{h} J^{+}=J^{+}(\widetilde{r}(h))
$$

where $\widetilde{r}(h) \in S_{+}^{-2,2}\left(\varepsilon, d, R_{1}\right)$ and

$$
\widetilde{r}(x, \xi ; h)=O\left(h^{\infty}\langle x\rangle^{-\infty}\right) \text { on } \Omega_{+}\left(2 \varepsilon, 2 d, 2 R_{1}\right) .
$$

Since $\tau f\left(H_{0}^{h}\right) U_{0}(\tau, h)$ can be written as

$$
\tau f\left(H_{0}^{h}\right) U_{0}(\tau, h)=i f_{1}\left(H_{0}^{h}\right)\left[A, U_{0}(\tau, h)\right] f_{2}\left(H_{0}^{h}\right),
$$


where $f_{1}(\lambda)=\frac{1}{2 \lambda} f(\lambda)$ and $f_{2} \in C_{0}^{\infty}\left(\mathbf{R}^{+}\right), f_{2}=1$ on supp $f$, we can derive from (4.17) that

$$
\left.\left.\left\|\int_{0}^{t} B_{6}(\tau, h)\langle A\rangle^{-1} d \tau\right\| \leq C h, \quad h \in\right] 0, h_{0}\right],
$$

uniformly in $t$. Now from (4.14) and (4.18) to (4.20), it follows that

$$
\left\|\langle A\rangle \chi\left(H^{h}\right) W_{+}(t, h) f\left(H_{0}^{h}\right)\langle A\rangle^{-1}\right\| \leq C
$$

uniformly in $t$ and $h$. Similarly we can prove that

$$
\left\|\langle A\rangle^{-1} \chi\left(H^{h}\right) W_{+}(t, h) f\left(H^{h}\right)\langle A\rangle\right\| \leq C^{\prime}
$$

uniformly in $t$ and $h$. Equation (4.12) follows by interpolation.

Now let $\chi \in C_{0}^{\infty}(J)$. Take $\chi_{j} \in C_{0}^{\infty}(J), j=1,2$, such that $\chi_{1}=1$ on $\operatorname{supp} \chi$ and $\chi_{2}=1$ on $\operatorname{supp} \chi_{1}$. Put

$$
S(t, h)=\chi_{2}\left(H_{0}^{h}\right) W_{+}(t, h)^{*} \chi_{1}\left(H^{h}\right) W_{-}(-t, h) \chi\left(H_{0}^{h}\right) .
$$

By (4.11) and Lemma 4.2,

$$
\chi_{2}\left(H_{0}^{h}\right) S^{*}(h) A S(h) \chi\left(H_{0}^{h}\right) f=\lim _{t \rightarrow+\infty} S(t, h)^{*} A S(t, h) f
$$

for all $f \in \mathscr{D}(A)$. We can write

$$
\begin{aligned}
S(t, h)^{*} A S(t, h)=S\left(t_{0}, h\right)^{*} A S\left(t_{0}, h\right) & \\
+\int_{t_{0}}^{t}\left\{R_{1}(r)^{*} A S(r, h)\right. & +S(r, h)^{*} A R_{1}(r) \\
& \left.+R_{2}(r)^{*} A S(r, h)+S(r, h)^{*} A R_{2}(r)\right\} d r
\end{aligned}
$$

where

$$
\begin{aligned}
& R_{1}(t)=\chi_{2}\left(H_{0}^{h}\right) R_{+}(t, h)^{*} \chi_{1}\left(H^{h}\right) W_{-}(-t, h) \chi\left(H_{0}^{h}\right), \\
& R_{2}(t)=-\chi_{2}\left(H_{0}^{h}\right) W_{+}(t, h)^{*} \chi_{1}\left(H^{h}\right) R_{-}(-t, h) \chi\left(H_{0}^{h}\right), \quad \text { and } \\
& R_{ \pm}(t)=i h^{-1} U(t, h)^{*} J^{ \pm}\left(r_{ \pm}(h)\right) U_{0}(t, h) .
\end{aligned}
$$

Recall that $r_{ \pm}(h)=e^{-i \phi_{ \pm} / h}\left(H^{h}-|\xi|^{2}\right)\left(e^{-i \phi_{ \pm} / h} a_{ \pm}(h)\right)$ and $a_{+}(h)\left(a_{-}(h)\right.$, resp. $)$ is an outgoing (incoming, resp.) $h$-parametrix.

Proposition 4.3. Let $b \in S_{+}^{0} \cap S_{-}^{0}$. Assume that $\operatorname{supp} b \subset\left\{(x, \xi) \in R^{2 n}\right.$; $|\widehat{x} \cdot \widehat{\xi}| \leq 1-\sigma\}, \sigma>0$. Let $J=] a, b[$ be an interval of nontrapping energy. For $0<\varepsilon_{1} \ll \sigma, 0<d_{1} \ll a$, let $a_{ \pm}(h)$ be the parametrix constructed in $\Omega_{ \pm}\left(\varepsilon_{1}, d_{1}, R_{1}\right)$ as before. Then with the above notations, there exists $T_{0}>0$ such that for any $N \in \mathbf{N}$,

$$
\left\|A R_{j}(t, h) b(x, h D)\right\| \leq C_{N} h^{N}\langle t\rangle^{-N}, \quad \text { for } t>T_{0}, j=1,2 .
$$


Proof. Consider first the case $j=2$. By Lemma 4.2,

$$
\begin{aligned}
& \left\|A R_{2}(t, h) b(x, h D)\right\| \\
& \quad \leq C h^{-1}\left\|A \chi_{1}\left(H^{h}\right) U(t, h) J^{-}\left(r_{-}(h)\right) U_{0}(-t, h) \chi\left(H_{0}^{h}\right) b(x, h D)\right\| \\
& \quad \leq C h^{-1}\langle t\rangle\left\|J^{-}\left(\widetilde{r}_{-}(h)\right) U_{0}(-t, h) \chi\left(H_{0}^{h}\right) b(x, h D)\right\|
\end{aligned}
$$

where $\widetilde{r}_{-}(h)$ is determined by $A J^{-}\left(r_{-}(h)\right)=J^{-}\left(\widetilde{r}_{-}(h)\right)$. Consequently, $\widetilde{r}_{-}(h)$ $\in S_{+}^{0,1}\left(\varepsilon_{1}, d_{1}, R_{1}\right)$ and $\tilde{r}_{-}(\chi, \xi ; h)=O\left(h^{\infty}\langle x\rangle^{-\infty}\right)$ in $\Omega_{+}\left(2 \varepsilon_{1}, 2 d_{1}, 2 R_{1}\right)$. $\tilde{r}_{-}(h)$ can be decomposed as

$$
\widetilde{r}_{-}(h)=r_{1}(h)+r_{2}(h)+r_{3}(h)
$$

where $\operatorname{supp} r_{1}(h) \subset\left\{(x, \xi) ;|x| \leq \frac{7}{2} R_{1}\right\}, \operatorname{supp} r_{2}(h) \subset\left\{(x, \xi),-1+\varepsilon_{1}<\right.$ $\left.\widehat{x}, \widehat{\xi}<-1+3 \varepsilon_{1},|\xi|^{2} \geq 3 d_{1},|x| \geq 3 R_{1}\right\}$ and $r_{3}(h) \in S^{-\infty,+\infty}$. Since $3 \varepsilon_{1}<\sigma$, we derive from Corollary 2.2 (for $U_{0}(t, h)$ ) that there exists $T_{0}>0$ such that

$$
\left.\| J^{-}\left(\tilde{r}_{-}(h)\right) U_{0}(-t, h) \chi\left(H_{0}^{h}\right) b(x, h D)\right) \|=O\left(h^{\infty}\langle t\rangle^{-\infty}\right), \quad t>T_{0} .
$$

This proves (4.23) for $j=2$.

For $j=1$, one can write

$$
W_{-}(-t, h)=J^{-}\left(a_{-}(h)\right)-i h^{-1} \int_{0}^{t} U(s ; h) J^{-}\left(r_{-}(h)\right) U_{0}(-s, h) d s .
$$

By Corollary 2.2 and the construction of $r_{ \pm}(h)$, we can easily check that there is $T_{0}>0$ such that for any $m \geq 0$,

$$
\left\|A J^{+}\left(r_{+}(h)\right)^{*} U(t+s ; h) \chi_{1}\left(H^{h}\right) J^{-}\left(r_{-}(h)\right)\langle x\rangle^{m}\right\|=O\left(h^{\infty}\langle t+s\rangle^{-\infty}\right)
$$

for $t+s>T_{0}$. From (4.25), it follows that

$$
\begin{gathered}
\left\|A J^{+}\left(r_{+}(h)\right)^{*} \int_{0}^{t} U(t+s ; h) \chi_{i}\left(H^{h}\right) J^{-}\left(r_{-}(h)\right) U_{0}(-s, h) \chi\left(H_{0}^{h}\right) b(x, h D) d s\right\| \\
=O\left(h^{\infty}\langle t\rangle^{-\infty}\right)
\end{gathered}
$$

for $t>T_{0}$. Since $b \in S_{+}^{0}\left(\sigma, d_{2}, R_{2}\right)$, choosing a larger $R_{1}=R_{1}\left(\varepsilon_{1}, d_{1}\right)$ if necessary (see (3.8)), we can prove by the calculus of Fourier integral operators that

$$
J^{-}\left(a_{-}(h)\right) b(x, h D)=\widetilde{b}(x, h D ; h) J^{-}(\widetilde{a}(h))+J^{-}(\widetilde{r}(h))
$$

where $\tilde{r}(h) \in S^{-\infty, \infty}, \tilde{a}(h) \in S^{0,0}$, and $\widetilde{b}(h) \in S_{+}^{0,0} \cap S_{-}^{0,0}$ with support contained in $\{|\widehat{x}, \widehat{\xi}|<1-\sigma / 2\}$. Therefore by the construction of $r_{+}(h)$, there exists some $T_{0}>0$ such that

$$
\begin{aligned}
& \left\|A J^{+}\left(r_{+}(h)\right)^{*} U(t, h) \chi_{1}\left(H^{h}\right) J^{-}\left(a_{-}(h)\right) \chi\left(H_{0}^{h}\right) b(x, h D)\right\| \\
& \quad \leq O\left(h^{\infty}\langle t\rangle^{-\infty}\right)+C\left\|A J^{+}\left(r_{+}(h)\right)^{*} U(t, h) \chi_{1}\left(H^{h}\right) b(x, h D ; h)\right\| \\
& \quad \leq O\left(h^{\infty}\langle t\rangle^{-\infty}\right), \quad t>T_{0} .
\end{aligned}
$$


From (4.24), (4.26), and (4.27), it results that:

$$
\begin{aligned}
& \left\|A R_{1}(t) b(x, h D)\right\| \\
& \quad \leq C\langle t\rangle\left\|A J^{+}\left(r_{+}(h)\right)^{*} U(t, h) \chi_{1}\left(H^{h}\right) W_{-}(t, h) \chi_{2}\left(H_{0}^{h}\right) b(x, h D)\right\| \\
& \quad \leq O\left(h^{\infty}\langle t\rangle^{-\infty}\right)
\end{aligned}
$$

for $t>T_{0}$. This finishes the proof of Proposition 4.3.

Now since $T(h) f\left(H_{0}^{h}\right)=f_{1}\left(H_{0}^{h}\right)\left\{A-S(h)^{*} A S(h)\right\} \chi\left(H_{0}^{h}\right)$, where $f_{1}(\lambda)=$ $\frac{1}{2 \lambda} f(\lambda)$ and $\chi(\lambda)=1$ for $\lambda \in \operatorname{supp} f$, it follows from (4.21) and (4.22) and Proposition 4.3 that the following result holds.

Corollary 4.4. Let $J$ be an interval of nontrapping energy, $f \in C_{0}^{\infty}(J)$. Assume $a, b \in S_{+}^{0} \cap S_{-}^{0}$. Then there exists some $T_{0}>0$ such that

$$
\begin{aligned}
\| a(x, h D)\{T(h) & f\left(H_{0}^{h}\right)-f_{1}\left(H_{0}^{h}\right) \\
& \left.\times\left(A \chi\left(H_{0}^{h}\right)-S(t, h)\right)^{*} A S(t, h)\right\} b(x, h D) \|=O\left(h^{\infty}\right)
\end{aligned}
$$

uniformly in $t>T_{0}$. Here $S(t, h)$ is defined as in (4.21). In particular, $a(x, h D) T(h) f\left(H_{0}^{h}\right) b(x, h D)$ is an h-pseudodifferential operator.

Proof. By the calculus of Fourier integral operators (see, e.g., [25, Appendix]) and the semiclassical Egorov theorem (see Theorem A, Appendix), one sees clearly that $S(t, h)^{*} A S(t, h)$ is an $h$-pseudodifferential operator. Equation (4.28) follows from Proposition 4.3. From this it is also clear that the remainder in equation (4.28) is in fact a smoothing operator. So it follows that $a(x, h D) T(h) f\left(H_{0}^{h}\right) b(x, h D)$ is an $h$-pseudodifferential operator with symbols equal to that of $a(x, h D) f_{1}\left(H_{0}^{h}\right)\left(A \chi\left(H_{0}^{h}\right)-S(t, h)^{*} A S(t, h)\right) b(x, h D)$ up to an error of order $O\left(h^{\infty}\right)$.

In the following we shall compute the $h$-principal symbol of

$$
a(x, h D) f\left(H_{0}^{h}\right) T(h) b(x, h D) .
$$

Our computation is based on the results of Lemmas 3.2 and 3.3.

Proposition 4.5. For $b \in S^{m}, a_{j} \in S_{+}^{0} \cap S_{-}^{0}$, there exists $t_{0}>0$ such that for $t>t_{0}$,

$$
a_{1}(x, h D) W_{-}(-t, h)^{*} b\left(x_{1} h D\right) W_{-}(-t, h) a_{2}(x, h D)=C(x, h D ; h) .
$$

Here $C(h) \in S^{m, 0}$ admits an asymptotic expansion: $C(h) \sim \sum_{j=0}^{\infty} h^{j} C_{j}$ with $C_{0}$ given by

$$
C_{0}(x, \xi)=a_{1}(x, \xi) a_{2}(s, \xi) b\left(\Omega_{-}^{\mathrm{cl}}(x, \xi)\right) .
$$

$\Omega_{-}^{\mathrm{cl}}$ is the classical incoming wave operator.

Proof. Recall first that if $d_{j} \in S^{m j}, j=1,2$, then

$$
J^{-}\left(d_{1}\right)^{*} J^{-}\left(d_{2}\right)=e(x, h D ; h)
$$


is an $h$-pseudodifferential operator with symbol $e(h) \in S^{m_{1}+m_{2}, 0}$. In addition, $e(h) \sim \sum_{j=0}^{\infty} h^{j} e_{j}$ and $e_{0}$ is given by

$$
e_{0}(x, \xi)=d_{1}\left(y_{-}(x, \xi), \xi\right) d_{2}\left(y_{-}(x, \xi), \xi\right)\left|\frac{\partial y_{-}(x, \xi)}{\partial x}\right| .
$$

By the Egorov theorem, $U(t, h)^{*} b(x, h D) U(t, h)$ is an $h$-pseudodifferential operator with $h$-principal symbol $b\left(\phi^{t}(x, \xi)\right)$, where $\phi^{t}=(q(t), p(t))$ is the Hamiltonian flow. From (4.29) one sees that

$$
a_{1}(x, h D) W_{-}(-t, h)^{*} b(x, h D) W_{-}(-t, h) a_{2}(\chi, h D)
$$

is an $h$-pseudodifferential operator with $h$-principal symbol $C_{0}$ :

$$
C_{0}(x, \xi)=a_{1}(x, \xi) a_{2}(x, \xi) d(x-2 t \xi, \xi),
$$

where

$$
\begin{aligned}
& d(x, \xi)=a_{0,-}\left(y_{-}(x, \xi), \xi\right)^{2}\left|\frac{\partial y_{-}}{\partial x}(x, \xi)\right| d_{1}(x, \xi), \\
& d_{1}(x, \xi)=b\left(\phi^{t}\left(y_{-}(x, \xi)\right), \nabla_{x} \phi_{-}\left(y_{-}(x, \xi), \xi\right)\right) .
\end{aligned}
$$

For $(x, \xi) \in \operatorname{supp} a_{1}$, one has

$$
|x-2 t \xi| \geq \delta(|x|+2 t|\xi|), \quad \delta>0, \forall t>0 .
$$

Since $y_{-}(x, \xi)-x=O\left(\langle x\rangle^{-\varepsilon_{0}}\right)$, there exists some $t_{0}>0$ such that $(x, \xi) \in$ $\operatorname{supp} a_{1}$ implies that $\left(y_{-}(x-2 t \xi, \xi), \xi\right) \in \Omega_{-}\left(2 \varepsilon_{1}, 2 d_{1}, 2 R_{1}\right)$ for $t>t_{0}$. According to Lemma 3.3 , for $\left(y_{-}, \xi\right) \in \Omega_{-}\left(2 \varepsilon_{1}, 2 d_{1}, 2 R_{1}\right), a_{0}\left(y_{-}, \xi\right)^{2}\left|\partial y_{-} / \partial x\right|$ $=\left|\operatorname{det}\left(\partial_{x} \partial_{\xi} \phi_{-}\left(y_{-}, \xi\right)\right)\right|=1$, since $\partial_{\xi} \phi_{-}\left(y_{-}(x, \xi), \xi\right)=x$. Therefore for $t>t_{0}, C_{0}(x, \xi)=a_{1}(x, \xi) a_{2}(x, \xi) d_{1}(x-2 t \xi, \xi)$. Put $\phi_{0}^{t}(x, \xi)=(x+2 t \xi, \xi)$. From the definition of $\Omega_{-}^{\mathrm{cl}}(x, \xi)$ [16], one sees that

$$
\Omega_{-}^{\mathrm{cl}}(x, \xi)=\left(q_{-}(0 ; x, \xi), p_{-}(0 ; x, \xi)\right),
$$

where $\left(q_{-}(t), p_{-}(t)\right)$ is the solution of the Hamilton system (1.3) with the condition

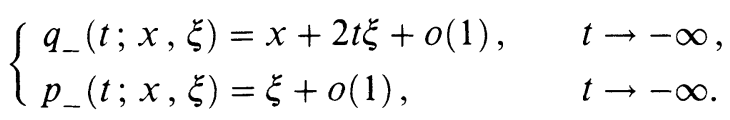

From the proof of Lemma 3.2, it is clear that for $(x, \xi) \in \Omega_{-}\left(2 \varepsilon_{1}, 2 d_{1}, 2 R_{1}\right)$, $R_{1}>0$ large enough, $q_{-}(t ; x, \xi)=q\left(t ; y_{-}(x, \xi), \nabla_{x} \phi_{-}\left(y_{-}(x, \xi), \xi\right)\right)$. This means

$$
\Omega_{-}^{\mathrm{cl}}(x, \xi)=\left(y_{-}(x, \xi), \nabla_{x} \phi_{-}\left(y_{-}(x, \xi), \xi\right)\right) \quad \text { for }(x, \xi) \in \Omega_{-}\left(2 \varepsilon_{1}, 2 d_{1}, 2 R_{1}\right) \text {. }
$$

But for $(x, \xi) \in \operatorname{supp} a_{1}, t>t_{0}$, one always has

$$
\phi_{0}^{-t}(x, \xi) \in \Omega_{-}\left(2 \varepsilon_{1}, 2 d_{1}, 2 R_{1}\right) .
$$

Therefore,

$$
d_{1}\left(\phi_{0}^{-t}(x, \xi)\right)=b\left(\phi^{t} \circ \Omega_{-}^{\mathrm{cl}} \circ \phi_{0}^{-t}(x, \xi)\right)=b\left(\Omega_{-}^{\mathrm{cl}}(x, \xi)\right) .
$$

In the last equality, we have used the intertwining property of classical wave operators. 
Proposition 4.6. With the notation of Corollary 4.4, for any $a, b \in S_{+}^{0} \cap S_{-}^{0}$, there is $t_{0}>0$ such that $a(x, h D) f_{1}\left(H_{0}^{h}\right)\left(A-S(t, h)^{*} A S(t, h)\right) b(x, h D)$ is an $h$-pseudodifferential operator of order zero in $x$ and that its symbol has an expansion of the form: $C(h) \sim \sum C_{j} h^{j}$, where $C_{j}$ is independent of $t>t_{0}$ for all $j \geq 0$ and

$$
C_{0}(x, \xi)=f\left(\xi^{2}\right) a(x, \xi) b(x, \xi) \tau \circ \Omega_{-}^{\mathrm{cl}}(x, \xi) .
$$

Here $\tau$ is the classical time-delay function.

Proof. Recall first that $\chi_{1}\left(H^{h}\right)$ is an $h$-pseudodifferential operator with $h$ principal symbol $\chi_{1}\left(|\xi|^{2}+V(x)\right)$. From Proposition 4.5, it follows immediately that $a(x, h D) f_{1}\left(H^{h}\right) S(t, h)^{*} A x S(t, h) b(x, h D)$ is an $h$-pseudodifferential operator and when $t>t_{0}$, its $h$-principal symbol is given by

$$
d(x, \xi)=a(x, \xi) b(x, \xi) f_{1}\left(|\xi|^{2}\right) F\left(\Omega_{-}^{\mathrm{cl}}(x, \xi)\right),
$$

where $F$ is the $h$-principal symbol of $W_{+}(t, h) \chi_{2}\left(H_{0}^{h}\right) A \chi_{2}\left(H_{0}^{h}\right) W_{+}(t, h)^{*}$;

$$
F(x, \xi)=G\left(\phi^{t}(x, \xi)\right)
$$

and

$$
\begin{aligned}
G(x, \xi)=a_{0,+}\left(x, \eta_{+}(x, \xi)\right)^{2}\left|\frac{\partial \eta_{+}}{\partial \xi}\right| & \left\{\nabla_{\xi} \phi_{+}\left(x, \eta_{+}(x, \xi)\right) \cdot \eta_{+}(x, \xi)\right. \\
& \left.-2 t\left|\eta_{+}(x, \xi)\right|^{2} \chi_{2}\left(\left|\eta_{+}(x, \xi)\right|^{2}\right)^{2}\right\} .
\end{aligned}
$$

By Lemma 3.2, $\eta_{+}(x, \xi)=\xi-\int_{0}^{+\infty} \nabla V(q(t ; x, \xi)) d t$ is invariant by $\phi^{t}$;

$$
\eta_{+}\left(\phi^{t}(x, \xi)\right)=\eta_{+}(x, \xi), \quad \forall t \in R,(x, \xi) \in \Omega_{+}\left(2 \varepsilon_{1}, 2 d_{1}, 2 R_{1}\right) .
$$

According to Lemma 3.3, for $(x, \xi) \in \Omega_{+}\left(2 \varepsilon_{1}, 2 d_{1}, 2 R_{1}\right)$, one has

$$
a_{0,+}\left(x, \eta_{+}(x, \xi)\right)^{2}\left|\frac{\partial \eta_{+}}{\partial \xi}\right|=1 \text {. }
$$

By a direct computation, we can check that

$$
\nabla_{\xi} \phi_{+}\left(q(t ; x, \xi), \eta_{+}(x, \xi)\right)=\nabla_{\xi} \phi_{+}\left(x, \eta_{+}(x, \xi)\right)+2 t \eta_{+}(x, \xi),
$$

for $(q(t), \xi) \in \Omega_{+}\left(2 \varepsilon_{1}, 2 d_{1}, 2 R_{1}\right)$ with possibly larger $R_{1}$. On the other hand, for $(x, \xi) \in \operatorname{supp} a$, one has $\widehat{x} \cdot \widehat{\xi}>-1+\sigma, \sigma \gg \varepsilon_{1}>0$. If $|x| \geq R_{2}$ with $R_{2}$ large enough, then by the expression for $\Omega_{-}^{\mathrm{cl}}$ :

$$
\Omega_{-}^{\mathrm{cl}}(x, \xi)=\left(y_{-}(x, \xi), \nabla_{x} \phi_{-}\left(y_{-}(x, \xi), \xi\right)\right),
$$

one can derive that

$$
\phi^{t}\left(\Omega_{-}^{\mathrm{cl}}(x, \xi)\right) \in \Omega_{+}\left(2 \varepsilon_{1}, 2 d_{1}, 2 R_{1}\right) \text { for all } t>0 .
$$

If $|x|<R_{2},|\xi|^{2} \in \operatorname{supp} f_{1}$, then $\left|\Omega_{-}^{\mathrm{cl}}(x, \xi)\right| \leq C$, where $C$ depends only on $R_{2}$ and $f_{1}$. By the nontrapping assumption, there exists $t_{0}>0$ such that 


$$
\phi^{t}\left(\Omega_{-}^{\mathrm{cl}}(x, \xi)\right) \in \Omega_{+}\left(2 \varepsilon_{1}, 2 d_{1}, 2 R_{1}\right), \quad t>t_{0} .
$$

Summing up, we have proved that there exists $t_{0}>0$ large enough, such that

$$
\phi^{t}\left(\Omega_{-}^{\mathrm{cl}}(x, \xi)\right) \in \Omega_{+}\left(2 \varepsilon_{1}, 2 d_{1}, 2 R_{1}\right)
$$

for $t>t_{0}$ and $(x, \xi) \in \operatorname{supp} a,|\xi|^{2} \in \operatorname{supp} f_{1}$. From the definition of $\tau$ and Lemma 3.2 , one see that

$$
\tau(x, \xi)=\frac{1}{2 p(x, \xi)}\left(\tau_{-}(x, \xi)-\tau_{+}(x, \xi)\right),
$$

where

(A) $\tau_{ \pm}(x, \xi)=\nabla_{\xi} \phi_{ \pm}\left(x, \eta_{ \pm}(x, \xi)\right) \cdot \eta_{ \pm}(x, \xi), \quad(x, \xi) \in \Omega_{ \pm}\left(2 \varepsilon_{1}, 2 d_{1}, 2 R_{1}\right)$.

Now from (4.30) and (A), it follows that

$$
d(x, \xi)=a(x, \xi) b(x, \xi) f_{1}\left(|\xi|^{2}\right) \tau_{+} \cdot \Omega_{-}^{\mathrm{cl}}(x, \xi) .
$$

Here we have used the relation $\left|\eta_{+}\left(\Omega_{-}^{\mathrm{cl}}(x, \xi)\right)\right|^{2}=|\xi|^{2}$ and $\chi_{2}=1$ on $\operatorname{supp} f_{1}$. Remark that $\tau_{ \pm}$is well defined on $p^{-1}(J), p(x, \xi)=|\xi|^{2}+V(x)$. For $|\xi|^{2} \in$ $J$, we can check directly that $\tau_{-} \cdot \Omega_{-}^{\mathrm{cl}}(x, \xi)=x \cdot \xi$. From (A) and (4.31), it results that for $t>t_{0}$, the $h$-principal symbol of

$$
a(x, h D) f_{1}\left(H_{0}^{h}\right)\left(A-S(t, h)^{*} A S(t, h)\right)^{*} b(x, h D)
$$

is $f\left(|\xi|^{2}\right) a(x, \xi) b(x, \xi) \tau \circ \Omega_{-}^{\mathrm{cl}}(x, \xi)$.

Now we are able to give easily the proof of Theorems 1 and 2.

Proof of Theorem 1. If $a, b \in S_{+}^{0} \cap S_{-}^{0}$, the results follow from (4.1), Corollary 4.4, and Proposition 4.6. If $a, b \in C_{0}^{\infty}\left(R_{x}^{n}\right)$, notice that by Corollary 2.2 for any $N \geq 0$, one has

$$
\left\|A(h) R_{j}(t, h) g(x)\right\| \leq C_{N} h^{N}\langle t\rangle^{-N}, \quad t>t_{0}, j=1,2 .
$$

Here $R_{j}(t, h)$ is defined in Proposition 4.3 and $g=a$ or $b$. From (4.22) it follows that $\left\|a(x)\left\{T(h) f\left(H_{0}^{h}\right)-f_{1}\left(H_{0}^{h}\right)\left(A-S(t, h)^{*} A S(t, h)\right)\right\} b(x)\right\|=O\left(h^{\infty}\right)$ uniformly in $t>t_{0}$. Now the desired results can be derived as in Proposition 4.6. The details are omitted.

Proof of Theorem 2. Recall that $\chi\left(\bar{H}_{0}^{h}\right) \bar{T}(h)$ is uniformly bounded in $\mathscr{L}\left(L^{2}\right)$ by the nontrapping assumption on $J$, (see [27]):

$$
\left.\left.\left\|\chi\left(\bar{H}_{0}^{h}\right) \bar{T}(h)\right\| \leq C<+\infty, \quad h \in\right] 0, h_{0}\right] .
$$

By an argument of density, it suffices to prove (1.9) for $f, g \in C_{0}^{\infty}\left(R^{n}\right)$. Now take $a \in C_{0}^{\infty}\left(R^{n}\right), a(x)=1$ for $x$ near $x_{0}$. Put

$$
f_{h}=U_{h} W_{h}\left(x_{0}, \xi_{0}\right) f, \quad g_{h}=U_{h} W_{h}\left(x_{0}, \xi_{0}\right) g
$$


Then for $h>0$ small enough, one has

$$
\left\langle W_{h}\left(x_{0}, \xi_{0}\right)^{*} \chi\left(\bar{H}_{0}^{h}\right) \bar{T}(h) W_{h}\left(x_{0}, \xi_{0}\right) f, g\right\rangle=\left\langle a T(h) \chi\left(H_{0}\right) a f_{h}, g_{h}\right\rangle .
$$

Now (1.9) follows from Theorem 1 by the arguments already used in [27]. See the proof of Theorem 6.3 in [27] and Theorem B in Appendix.

\section{APPENDIX. A SEMIClassical Egorov theOREM}

For the reader's convenience, we state here a semiclassical Egorov theorem used in this paper, which is a particular case of Theorem 3.7 in [22]. See also [17] when $b \in S^{0}$.

Theorem A. Let $b \in S^{m}, m \in R$. Then under the assumption (2.1) on $V, U(t, h)^{*} b(x, h D) U(t, h)$ is an $h-\psi$ DO with symbol $f(t, h) \in S^{m, 0}$. Moreover, $f(t, h) \sim \sum_{j=0}^{\infty} h^{j} f_{j}(t)$, where each $f_{j}(t)$ can be computed from $b$ and the Hamilton flow $\phi^{t}$ of (1.3). In particular, one has $\operatorname{supp} f_{j}(t) \subset \operatorname{supp} b\left(\phi^{t}\right)$, for all $j \geq 0$, and $f_{0}(t)=b\left(\phi^{t}\right)$.

From Theorem A we can easily derive the following result.

Theorem B. Let $b \in S^{m}$ and $U_{h}$ be defined as in Introduction. Then for any $\left(x_{0}, \xi_{0}\right) \in R^{2 n}$ and $f \in \mathscr{S}\left(R^{n}\right)$, one has

$$
\begin{gathered}
\lim _{h \rightarrow 0_{+}} W_{h}\left(x_{0}, \xi_{0}\right)^{*} U_{h}^{*} U(t, h)^{*} b(x, h D) U(t, h) U_{h} W_{h}\left(x_{0}, \xi_{0}\right) f \\
=b(q(t), p(t)) f, \quad \text { in } L^{2}\left(R^{n}\right) .
\end{gathered}
$$

Here $(q(t), p(t))$ is a solution to (1.3) with initial data $\left(x_{0}, \xi_{0}\right)$.

For the proof of Theorem B, one is referred to [22]. Notice that in [22] the results are proved for Weyl $\psi$ DOs. But there is a well-known equivalence between different quantifications [17].

Acknowledgment. This work is partially supported by Chinese National Science Foundation and by State Education Commission of China. The author also thanks the referee for his suggestions on the organization of this paper.

\section{REFERENCES}

1. W. O. Amrein and M. Cibils, Global and Eisenbud-Wigner time-dealy in scattering theory, Helv. Phys. Acta 60 (1987), 481-500.

2. W. O. Amrein, M. Cibils, and K. Sinha, Configuration space properties of the S-matrix and time-delay in potential scattering, Ann. Inst. H. Poincaré Sect. A (N.S.) 47 (1987), 367-382.

3. V. Enss, Asymptotic completeness for quantum mechanical potential scattering. I, Comm. Math. Phys. 61 (1978), 285-291.

4. B. Helffer and D. Robert, Calcul fonctionnel par la transformation de Mellin et opérateurs admissibles, J. Funct. Anal. 53 (1983), 246-268. 
5. H. Isozaki, Differentiability of generalized Fourier transform associated with Schrödinger operators, J. Math. Kyoto Univ. 25 (1985), 789-806.

6. H. Isozaki and H. Kitada, Modified wave operators with time-independent modifiers, J. Fac. Sci. Univ. Tokyo 32 (1985), 77-104.

7. __ A remark on the microlocal resolvent estimates for two-body Schrödinger operators, RIMS, Kyoto University, 1986.

8. A. Jensen, Propagation estimates for Schrodinger type operators, Trans. Amer. Math. Soc. 291 (1985), 129-144.

9. A. Jensen, E. Mourre and P. Perry, Multiple commutator estimates and resolvent smoothness in quantum scattering theory, Ann. Inst. H. Poincare 41A (1984), 207-225.

10. H. Kitada, A relation between the modified wave operators $W_{J}^{ \pm}$and $W_{D}^{ \pm}$, preprint 1986.

11. Ph. Martin, Time-delay of quantum scattering processes, Acta Phys. Austriaca Suppl. 23 (1981), 157-208.

12. V. P. Maslov and M. V. Fedoriuk, Semiclassical approximation in quantum mechanics, Reidel, Dordrecht, 1981.

13. S. Nakamura, Time-delay and Lavine's formula, Comm. Math. Phys. 109 (1987), 397-415.

14. H. Narnhofer, Time-delay and dilation properties in scattering theory, J. Math. Phys. 25 (1984), 987-991.

15. R. Newton, Scattering theory of waves and particles, texts and monographs in physics, $2 \mathrm{nd}$ ed., Springer-Verlag, 1982.

16. M. Reed and B. Simon, Methods of modern mathematical physics. III, Scattering theory, Academic Press, New York, 1979.

17. D. Robert, Autour de l'approximation semiclassique, Progr. Math., No. 68, Birkhäuser, 1987.

18. D. Robert and H. Tamura, Semiclassical asymptotics for spectral density and time-delay problem in scattering processes, J. Funct. Anal. 80 (1988), 124-147.

19. __ Asymptotic behavior of scattering amplitudes in semiclassical and low energy limits, to appear.

20. A. V. Sobolev and D. R. Yafaev, On the quasi-classical limit of the total scattering cross section in non relativistic quantum mechanics, Ann. Inst. H. Poincaré Sect. A (N.S.) 44 (1986), 195-210.

21. X. P. Wang, Etude semiclassique d'observables quantiques, Ann. Fac. Sci. Toulouse Math. 7 (1985), 101-135.

22. __ Approximation semiclassique de l'équation de Heisenberg, Comm. Math. Phys. 104 (1986), 77-86.

23. __ Phase space description of time-delay in scattering theory, Comm. Partial Differential Equations 13 (1988), 223-259.

24. __ Time-delay operator for a class of singular potentials, Helv. Phys. Acta 60 (1987), 501-509.

25. __ Time-delay of scattering solutions and resolvent estimates for semiclassical Schrodinger operators, J. Differential Equations 71 (1988), 248-296.

26. __ Time-delay of scattering solutions and classical trajectories, Ann. Inst. H. Poincaré Sect. A (N.S.) 47 (1987), 25-37.

27. __ Time-delay operators in semiclassical limit. I,_ Finite range potentials, Ann. Scuola Norm. Sup. Pisa Cl. Sci. (4) 15 (1988), 1-34.

28. K. Yajima, The quasi classical limit of scattering amplitude- $L^{2}$ approach for short range potentials, Japan J. Math. 13 (1987), 77-126.

Department of Mathematics, Peking University, 100871 Beijing, China

Fachbereich Mathematik, MA7-2, Technische Universität Berlin, D-1000 Berlin 12, FRG (Current address) 